\title{
Speech Coding in the Brain: Representation of Vowel Formants by Midbrain Neurons Tuned to Sound Fluctuations ${ }^{1,2,3}$
}

\author{
든aurel H. Carney, ${ }^{1}$ Tianhao Li, ${ }^{1}$ and Joyce M. McDonough ${ }^{2}$
}

DOI:http://dx.doi.org/10.1523/ENEURO.0004-15.2015

${ }^{1}$ Departments of Biomedical Engineering, and Neurobiology \& Anatomy, University of Rochester, Rochester, New York 14642, and '2Department of Linguistics, University of Rochester, Rochester, New York 14627-0096

\begin{abstract}
Current models for neural coding of vowels are typically based on linear descriptions of the auditory periphery, and fail at high sound levels and in background noise. These models rely on either auditory nerve discharge rates or phase locking to temporal fine structure. However, both discharge rates and phase locking saturate at moderate to high sound levels, and phase locking is degraded in the CNS at middle to high frequencies. The fact that speech intelligibility is robust over a wide range of sound levels is problematic for codes that deteriorate as the sound level increases. Additionally, a successful neural code must function for speech in background noise at levels that are tolerated by listeners. The model presented here resolves these problems, and incorporates several key response properties of the nonlinear auditory periphery, including saturation, synchrony capture, and phase locking to both fine structure and envelope temporal features. The model also includes the properties of the auditory midbrain, where discharge rates are tuned to amplitude fluctuation rates. The nonlinear peripheral response features create contrasts in the amplitudes of low-frequency neural rate fluctuations across the population. These patterns of fluctuations result in a response profile in the midbrain that encodes vowel formants over a wide range of levels and in background noise. The hypothesized code is supported by electrophysiological recordings from the inferior colliculus of awake rabbits. This model provides information for understanding the structure of cross-linguistic vowel spaces, and suggests strategies for automatic formant detection and speech enhancement for listeners with hearing loss.
\end{abstract}

Key words: auditory nerve; computational model; midbrain; modulation tuning; speech coding

\section{Significance Statement}

Encoding of speech sounds is the most important function of the human auditory system. Current models for neural coding of speech fail over the range of sound levels encountered in daily life and in background noise. The acoustic structure of vowels and the properties of auditory midbrain neurons that are tuned to low-frequency amplitude fluctuations suggest a neural code for the spectral peaks (called formants) that identify vowels. The proposed neural code for speech sounds is the first that is robust over a wide range of sound levels and in background noise. These results address classic problems in auditory neuroscience and linguistics, and suggest novel strategies for auditory prosthetics, automatic speech recognition, and speech enhancement for hearing aids and telephones. 


\section{Introduction}

Vowels carry a heavy functional load in all languages, especially in running speech and discourse. How vowels are encoded by the nervous system across the range of sound levels important for vocal communication is unknown. The acoustic signature of vowels includes periodicity at the fundamental frequency ( $\mathrm{FO}$, or voice pitch); the harmonics of FO; and formants, the amplitude bands in the spectrum that characterize vowel contrasts (Fant, 1960). The first two formants are most important for vowel identification. Studies of auditory nerve (AN) speech coding typically focus on response rates or temporal synchrony at frequencies to which a fiber is most sensitive (Sachs and Young, 1979; Young and Sachs, 1979; Delgutte and Kiang, 1984; Schilling et al., 1998). These codes are adequate for low-level speech sounds in quiet, but they fail for moderate-to-high sound levels and in background noise. Vowels also induce systematic changes in the amplitude of F0-related fluctuations in AN responses. The vowel-coding hypothesis tested here focuses on the F0-related neural fluctuations and on contrasts in their amplitudes across neurons tuned to different frequencies.

Many inferior colliculus (IC) neurons display both spectral tuning, described by a most sensitive best frequency $(\mathrm{BF})$, and tuning to the frequency of sinusoidal fluctuations in amplitude, described by a best modulation frequency (BMF; Krishna and Semple, 2000; Joris et al., 2004; Nelson and Carney, 2007). Most IC neurons tuned for amplitude fluctuations have BMFs in the range of voice pitch (Langner, 1992) and are thus well suited to represent the critical acoustic features of vowels (Delgutte et al., 1998). The vowel-coding hypothesis presented here takes advantage of nonlinear properties of AN responses, including rate saturation (Sachs and Abbas, 1974; Yates, 1990; Yates et al., 1990) and synchrony capture, which is the dominance of a single stimulus frequency component on the response (Fig. 1; Young and Sachs, 1979; Deng and Geisler, 1987; Miller et al., 1997). These nonlinearities have strong effects on the rate fluctuations of AN fibers in response to vowels and provide a robust framework for encoding vowel features.

Received January 13, 2015; accepted June 18, 2015; First published July 02, 2015.

${ }^{1}$ The authors declare no competing financial interests.

${ }^{2}$ Author Contributions: L.H.C., T.L., and J.M.M. developed the model and wrote the paper; L.H.C. and T.L. performed the physiological experiments and analyzed the data

${ }^{3}$ This research was supported by National Institutes of Health-National Institute on Deafness and Other Communication Disorders Grant R01-001641.

T. Li's present address: Department of Electrical and Control Engineering, Liaoning Technical University, HuLuDao, LiaoNing 123000, People's Republic of China.

Acknowledgments: We thank Drs. David A. Cameron and Kenneth S. Henry for providing valuable comments on the manuscript.

Correspondence should be addressed to: Laurel H. Carney, Departments of Biomedical Engineering and Neurobiology \& Anatomy, University of Rochester, 601 Elmwood Avenue, Box 603, Rochester, NY 14642. E-mail: laurel.carney@rochester.edu.

DOI:http://dx.doi.org/10.1523/ENEURO.0004-15.2015

Copyright (C) 2015 Carney et al.

This is an open-access article distributed under the terms of the Creative Commons Attribution 4.0 International, which permits unrestricted use, distribution and reproduction in any medium provided that the original work is properly attributed.
Figure 1 introduces the main features of the vowel-coding hypothesis. The harmonic structure of vowels (Fig. 1A) yields two types of periodicity that coexist in AN responses, as follows: phase locking to harmonics near the $\mathrm{BF}$ of the fiber; and phase locking to slow fluctuations at F0. Fibers tuned near formant peaks may be saturated, and these fibers also have sustained responses that are dominated by phase locking to a single harmonic near the $\mathrm{BF}$ of the fiber, which is referred to as synchrony capture. Both of these nonlinearities result in responses with relatively weak fluctuations at FO (Fig. 1B). Fibers tuned to frequencies away from formants are not dominated by one harmonic but are influenced by the beating of multiple harmonics, resulting in strong low-frequency neural fluctuations at FO (Fig. 1C).

The contrast in the amplitude of low-frequency rate fluctuations across the AN population is enhanced in the midbrain by the rate tuning of IC neurons to amplitude modulations, which is described by modulation transfer functions (MTFs; Fig. 1D,E). The majority of MTFs in the IC have bandpass (BP) tuning to amplitude modulations (Fig. 1D), and the rest have band-reject tuning (Fig. 1E), lowpass or high-pass tuning (Nelson and Carney, 2007), or more complex MTFs that combine excitation and inhibition (Krishna and Semple, 2000). Midbrain cells with bandpass MTFs that have maxima (i.e., BMFs) near F0 are hypothesized to display decreased rates when the BF of the cell is near a formant frequency (Fig. 1F, green arrow) because the neural inputs have weak low-frequency rate fluctuations (Fig. 1B). Cells with bandpass MTFs but with BF between formants are hypothesized to have increased rates (Fig. 1F, orange arrow) because their neural inputs have strong low-frequency fluctuations (Fig. 1C). In contrast, cells with band-reject or low-pass MTFs and minima near F0 will have increased rates when formant frequencies are near the BF (Fig. 1G, green arrow), because their neural inputs have weak low-frequency fluctuations (Fig. 1B). Band-reject or low-pass cells will have decreased rates (Fig. 1G, orange arrow) when the BF is between formants and the inputs have strong fluctuations (Fig. 1C).

The contrast across frequency in the F0-related neural fluctuations sets up a code for formants that is translated into rate profiles in the midbrain (Fig. $1 F, G$ ). This study used computational models for AN fibers and IC neurons to explore the robustness of this proposed code across a wide range of sound levels and in background noise. Examples of recordings from IC neurons in awake rabbits support the model for cells that have simple bandpass or band-reject amplitude modulation tuning.

\section{Materials and Methods}

\section{Modeling}

A phenomenological model of AN responses that includes several key nonlinearities, including rate saturation, adaptation, and synchrony capture (Zilany et al., 2009, 2014) provided the inputs to the models for two types of midbrain neurons (Fig. 2A). IC cells with BP MTFs were simulated using the same-frequency inhibition-excitation (SFIE) model (Nelson and Carney, 2004), which explains tuning for the amplitude modulation frequency by the 


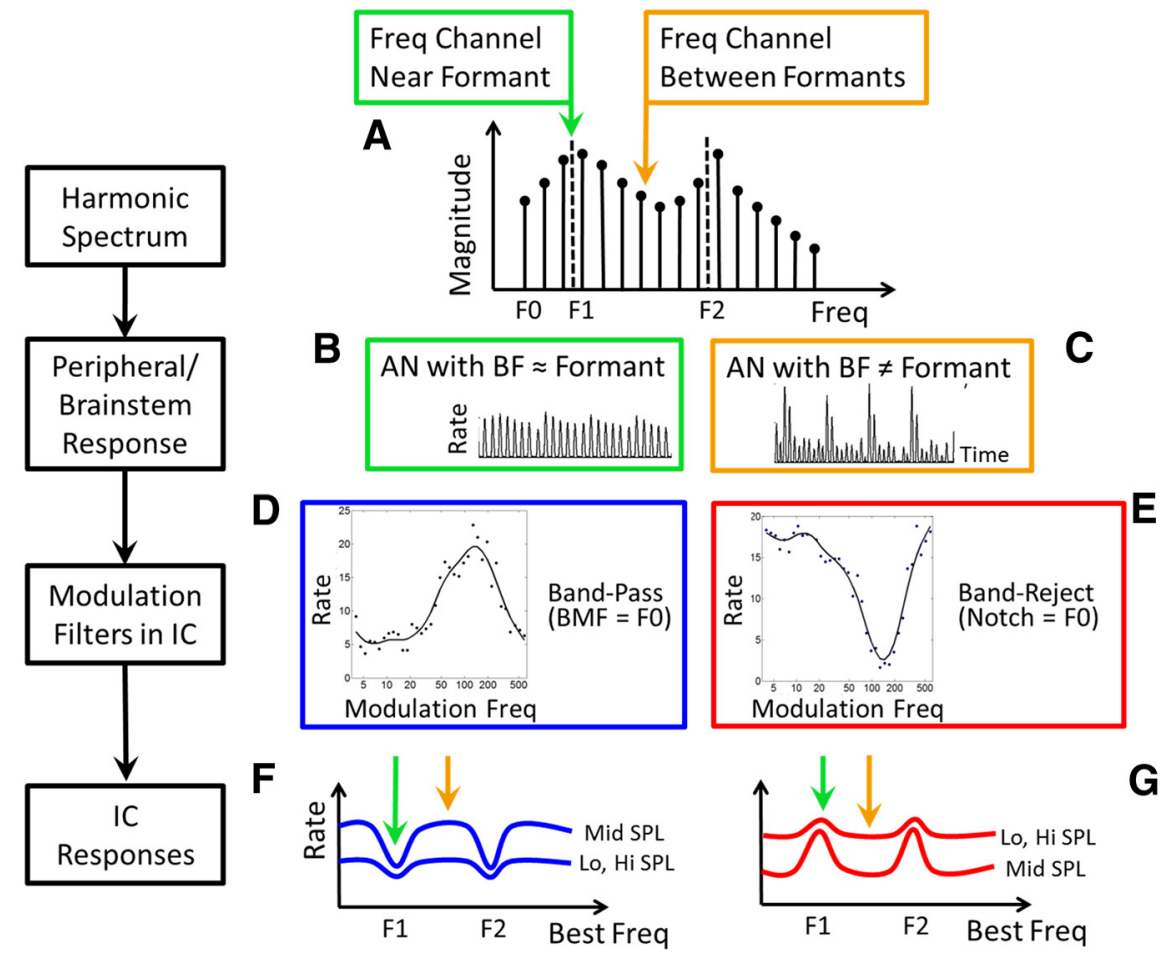

Figure 1 Schematic illustration of vowel-coding hypothesis. The left-hand column labels the key stages in the coding scheme. $\boldsymbol{A}$, Vowel spectrum consisting of harmonics of F0, shaped by the spectral envelope. B, Responses of AN fibers tuned near formants have relatively small pitch-related rate fluctuations. These responses are dominated by a single harmonic in the stimulus, referred to as synchrony capture. C, Fibers tuned between formants have strong rate fluctuations at F0 (Delgutte and Kiang, 1984). $\boldsymbol{D}$, Example of a bandpass MTF from rabbit IC with a BMF near F0 for a typical male human speaker. $\boldsymbol{E}$, Example band-reject MTF with a notch near a typical F0. $\boldsymbol{F}$, Bandpass midbrain neurons have reduced rates in frequency channels with weak fluctuations (green arrow) and increased rates in channels with strong fluctuations (see $\boldsymbol{C}$, orange arrow); thus dips in the rate profile of bandpass neurons encode F1 and F2. G, The profile of rates across a population of band-reject neurons has peaks at F1 and F2, because band-reject neurons respond more strongly to stimuli that result in reduced neural fluctuations in their inputs (see $\boldsymbol{B}$, green arrow).

interaction of excitatory and inhibitory inputs with different dynamics. IC cells with low-pass, band-reject (LPBR), or high-pass MTFs were simulated using an extension of the SFIE model; the LPBR model received excitatory input from the brainstem and inhibitory input from bandpass cells (Fig. 2B). Time-varying input rate functions to each model cell were convolved with $\alpha$ functions representing excitatory or inhibitory postsynaptic responses. The decay time constants of the $\alpha$ functions and the delays associated with synaptic responses were varied to produce MTFs tuned to different amplitude modulation frequencies (Nelson and Carney, 2004).

The parameter values for the cochlear nucleus (CN)/ brainstem neurons (Fig. 2) were fixed for all simulations. These parameters were the time constants of the excitatory and inhibitory $\alpha$ functions, $\tau_{\mathrm{CNex}}$ and $\tau_{\mathrm{CNinh}}$; the delay of the inhibitory $\alpha$ function with respect to the excitatory $\alpha$ function, $\mathrm{D}_{\mathrm{CNinh}}$; and amplitude scalars for the excitatory and inhibitory $\alpha$ functions, $\mathrm{A}_{\mathrm{CNex}}$ and $\mathrm{A}_{\mathrm{CNinh}}$ (for parameter values, see Table 1). These parameters resulted in "generic" cochlear nucleus or other brainstem neurons that project to the IC. In general, many types of $\mathrm{CN} /$ brainstem neurons have an increased synchrony to the stimulus envelope and a weak rate tuning to the amplitude modulation rate (Frisina et al., 1990; Grothe et al., 1997; Joris and Yin, 1998; Gai and Carney, 2008; for review, see Joris et al., 2004). The model CN/brainstem cells do not have significantly greater contrast in the rate versus $B F$ profiles in response to vowels than do AN fibers, thus this stage of the model is not an attempt to simulate the CN "chopper" neurons described by Blackburn and Sachs (1990), which have weaker temporal representations of vowels than AN fibers or other $\mathrm{CN}$ response types but more robust rate versus $B F$ profiles. The detailed response properties and connections of different types of $\mathrm{CN}$ and other brainstem neurons were not included in the simulations here.

Parameter values for model IC neurons are provided in Table 1. For model bandpass neurons, there were the following four parameters: the time constant of the excitatory $\alpha$ function, $\tau_{\mathrm{BPex}}$; the time constant of the inhibitory $\alpha$ function, $\tau_{\mathrm{BPinh}}$; the delay of the inhibition with respect to the excitation, $D_{\text {Bpinh }}$; and amplitude scalars for the excitatory and inhibitory inputs, $A_{B P e x}$ and $A_{B P i n h}$. These parameters were based on example model neurons with a range of BMFs in the study by Nelson and Carney (2004). Model band-reject, low-pass, and high-pass neurons (Fig. 2B) were described by the bandpass model parameters, plus the time constant of the excitatory $\alpha$ function, $\tau_{\text {LPBRex }}$; the time constant of the inhibitory $\alpha$ function, $\tau_{\text {LPBRinh}}$; the delay of the inhibition with respect to excita- 

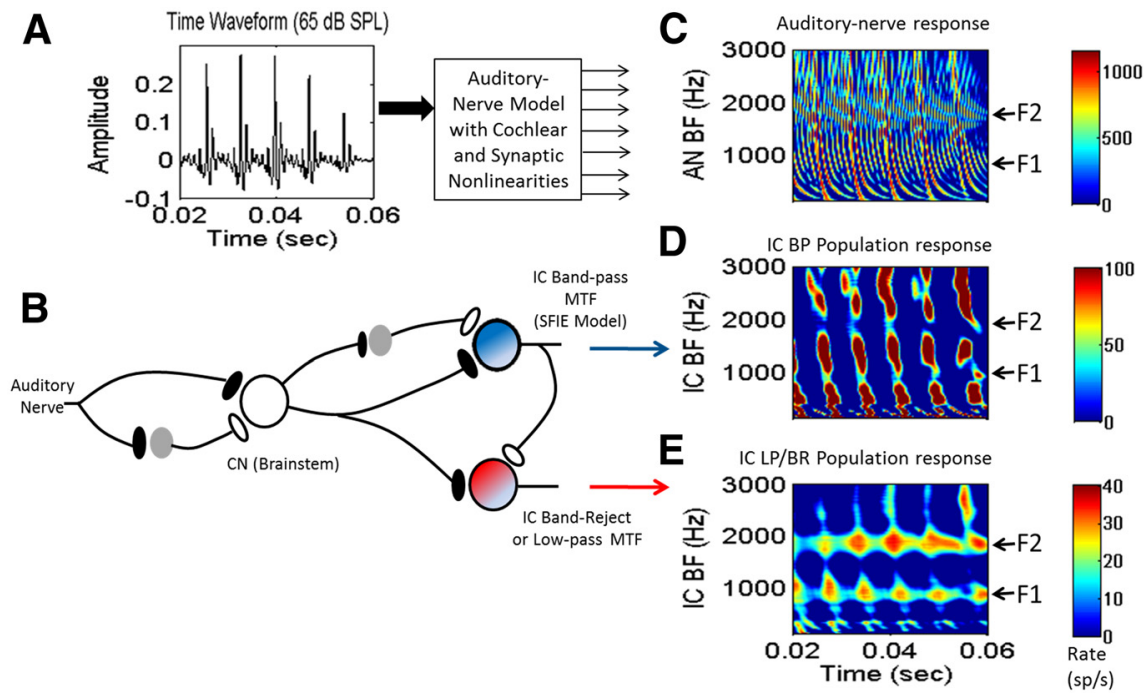

Figure 2 Models for modulation tuning in IC cells. A, Time waveform of the vowel /æ/ (had). B, The SFIE model (Nelson and Carney, 2004) for midbrain cells with BP MTFs (blue cell). An extension of the SFIE model is illustrated by the red cell, which is excited by ascending inputs and inhibited by the bandpass SFIE cell. This model cell simulates the relatively common low-pass or band-reject MTFs (see Fig. 3), and is referred to as the LPBR model. C, Model AN population response (Zilany et al., 2009, 2014). D, Population response of the BP IC model; BP neurons with BFs near F1 and F2 (arrows at right) have decreased responses (Fig. 1F). $E$, The LPBR model has peaks in the population rate profile near F1 and F2 (Fig. 1G).

tion, $\mathrm{D}_{\text {LPBRinh }}$; and amplitude scalars for the excitatory and inhibitory inputs, $A_{L P B R e x}$ and $A_{L P B R i n h}$.

For all models, the synaptic output signal from the auditory nerve model (which has units of spikes per

Table 1 Model parameters

\begin{tabular}{ll}
\hline \multicolumn{2}{c}{ CN/brainstem parameters (all simulations) } \\
\hline$\tau_{\text {ex }}(\mathrm{ms})$ & 0.5 \\
$\tau_{\text {inh }}(\mathrm{ms})$ & 2 \\
$D(\mathrm{~ms})$ & 1 \\
$\mathrm{~A}_{\text {ex }}$ & 1.5 \\
$\mathrm{~A}_{\text {inh }}$ & 0.9 \\
\hline
\end{tabular}

IC model parameters

\begin{tabular}{llll}
\hline Bandpass model & Fig. 3A & Fig. 3B & Fig. 3C \\
\hline$\tau_{\text {BPex }}(\mathrm{ms})$ & 2 & 0.7 & 5 \\
$\tau_{\text {BPinh }}(\mathrm{ms})$ & 6 & 0.7 & 10 \\
$\mathrm{D}_{\text {BP }}(\mathrm{ms})$ & 2 & 1.4 & 2 \\
$A_{\text {BPex }}$ & 2 & 3 & 6 \\
$A_{\text {BPinh }}$ & 2.2 & 4.2 & 6.6 \\
\hline Low-pass/band-reject model & Fig. 3A & Fig. 3B & Fig. 3C \\
\hline$\tau_{\text {LPBRex }}(\mathrm{ms})$ & 2 & 0.7 & 5 \\
$\tau_{\text {LPBRinh }}(\mathrm{ms})$ & 5 & 5 & 5 \\
$\mathrm{D}_{\text {LPBR }}(\mathrm{ms})$ & 0.7 & 0.7 & 0.7 \\
$A_{\text {LPBRex }}$ & 0.6 & 1 & 0.6 \\
$A_{\text {LPBRinh }}$ & 2 & 2 & 2
\end{tabular}

A single set of parameters was specified for the CN/brainstem level of the model. Three sets of parameters were used for the IC models, illustrated in Figure 3, which had BMFs of $45 \mathrm{~Hz}$ (left), $125 \mathrm{~Hz}$ (middle), and $16 \mathrm{~Hz}$ (right). The model parameters for other figures were the same as those for Figure $3 \mathrm{~B}$, which had a BMF near F0 for most of the vowels used as stimuli. second) was convolved with excitatory and inhibitory postsynaptic potentials for the $\mathrm{CN} /$ brainstem model. These potentials were modeled by $\alpha$ functions, each described by a time constant, and each normalized to have unit area before scaling the amplitudes with the coefficients described above. The model cell output was computed by subtracting the inhibitory signal from the excitatory potential and then half-wave rectifying. This model output signal was then convolved with the appropriate $\alpha$ function to provide the input to the next model cell, and excitatory and inhibitory signals were summed and half-wave rectified to compute the model IC response.

The basic properties of the model responses to the vowel /æ/ (in "had") are illustrated in Figure $2 \mathrm{C}$ as a function of time for a population of neurons tuned to a range of BFs (the frequency that elicits the strongest response). As illustrated schematically in Figure 1, the model AN fibers (Fig. 2C) tuned near formant frequencies (arrows at right) have strong response rates with relatively small F0-related fluctuations, and those tuned to intermediate frequencies have strong fluctuations in rate associated with each pitch period. A population of model BP cells with the MTF tuned to FO and a range of BFs is illustrated in Figure 2D. BP cells with BFs tuned to formant frequencies have weak responses compared to those tuned to intermediate frequencies, where the strong F0related fluctuations elicit strong responses (Figs. 1C, 2D). In contrast, model LPBR cells (Fig. 2E) with a notch in the MTF near F0 (Fig. 1E) respond best when the BF is tuned near a formant frequency; these cells respond weakly to the strong F0-related fluctuations on their inputs (Fig. 1C), and are more effectively driven by the weaker modula- 

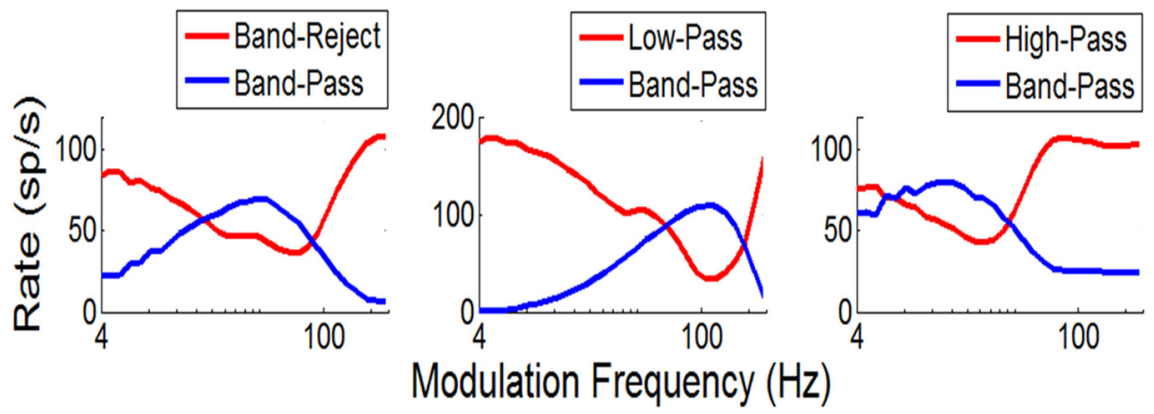

Figure $3 \boldsymbol{A}-\boldsymbol{C}$, Three bandpass MTFs (blue, as in Fig. 2) with mid-frequency $(\boldsymbol{A})$, high-frequency $(\boldsymbol{B})$, and low-frequency $(\boldsymbol{C})$ BMFs. MTFs for three model cells (red, as in Fig. 2) that are inhibited by the bandpass cells explain three other MTF types in the IC: the more common band-reject $(\boldsymbol{A})$ and low-pass $(\boldsymbol{B})$ MTFs, as well as the less common high-pass MTF $(\boldsymbol{C})$. Model parameters are in Table 1.

tions provided by the auditory periphery at the formant frequencies (Fig. 1B).

Figure 3 illustrates MTFs for three model BP neurons (blue curves) tuned to different amplitude modulation rates. Each of these model BP neurons provided an inhibitory input to an LPBR neuron, yielding the other set of MTFs (red curves). The shapes of these MTFs are characteristic of MTF types referred to as band reject, low pass, and high pass (Krishna and Semple, 2000; Nelson and Carney, 2007). This relatively simple model for modulation frequency tuning explains several of the MTF types that are encountered in the IC. Other IC cells have more complex MTFs, consisting of combinations of excitatory and inhibitory regions (Krishna and Semple, 2000). Further extensions of this modeling approach will be required to describe those MTFs.

\section{Physiological methods}

All animal procedures were performed in accordance with the regulations of the University of Rochester animal care committee. Recordings were made in the central nucleus of the IC in female awake rabbits using implanted tetrodes advanced through the $\mathrm{IC}$ with a head-mounted microdrive (5-Drive, Neuralynx). Tetrodes were constructed by twisting four strands of 12- or 18- $\mu \mathrm{m}$-diameter epoxy-coated platinum iridium wire. Action potentials were sorted offline based on spike shapes (Schwarz et al., 2012). Singleunit recordings were identified based on a criterion of $<2 \%$ for interspike intervals $<1 \mathrm{~ms}$ and, when multiple spikes were recorded and sorted, on values $<0.1$ of the summed cluster separation metric $\left(L_{\Sigma}\right.$, based on a sum of the cluster isolation metric $L_{\text {ratio }}$, from Schmitzer-Torbert et al., 2005; Schwarz et al., 2012).

Acoustic stimuli were created in Matlab and presented using Tucker-Davis Technologies hardware and Beyer Dynamic headphones through custom earmolds. Stimuli were calibrated using an Etymotic ER-7C probe-tube microphone. Audio frequency tuning was determined using response maps based on responses to $200 \mathrm{~ms}$ tones presented every $600 \mathrm{~ms}$ with frequencies from 0.2 to 20 $\mathrm{kHz}$ and levels from 10 to $70 \mathrm{~dB}$ SPL, presented in random order. Amplitude modulation tuning was determined using $100 \%$ modulated wideband noise (30 dB SPL spectrum) or tone carriers (70 dB SPL) near the best frequencies of the neurons. Vowel stimuli (65 dB SPL) were from the database of Hillenbrand et al. (1995). Samples were extracted from the steady-state portion of the vowel, and a Hanning window was applied to limit the duration to $200 \mathrm{~ms}$. Vowel stimuli were chosen from the database based on the match of the speaker's average FO to the BMF of the neuron.

\section{Results}

\section{Model responses}

Responses of model IC cells with bandpass and bandreject MTFs to two vowel tokens (Fig. 4) illustrate the representation of formants in the average rate profiles of model IC population responses. As schematized in Fig. 1, the profile of average discharge rates for a population of model IC neurons with BP MTFs (Fig. 4C,D, blue) has minima at BFs near the vowel formants. In contrast, the rates of band-reject neurons (Fig. 4C,D, red) have peaks at the formants. The importance of the LPBR model for a robust neural code of vowel formants is illustrated in Figure 4D for the vowel /i/, which, like many vowels, has widely spaced formants. This response shows that reductions in the discharge rate of BP responses (Fig. 4D, blue) are ambiguous, as they may be due either to reduced fluctuations of AN responses tuned near formants (Fig. 1B) or to reduced spectral energy (Fig. 4D, arrow, 1500 $\mathrm{Hz}$ ). This ambiguity is resolved by the LPBR model (Fig. $4 D$, red), which only responds when both sufficient energy and reduced fluctuations are present on the inputs to the model midbrain cell. The reduced fluctuations result in the disinhibition of the LPBR model by reducing the inhibitory input from the BP neuron. Note that the model LPBR population rate profile is qualitatively similar to the $A N$ (Fig. 4C,D, magenta) and CN/brainstem (Fig. 4C,D, cyan) profiles, except that the LPBR population responses (Fig. $4 C, D$, red curves) have greater contrast and steeper slopes in the discharge rates across the population in comparison with the peripheral responses. The LPBR model average rate profiles differ from peripheral rate profiles in being more robust for vowels in background noise and across a wide range of sound levels (see below).

The midbrain vowel-coding hypothesis is robust across a wide range of SPLs (Fig. 5) because it is based on the pattern of pitch-related rate fluctuations in AN responses, 

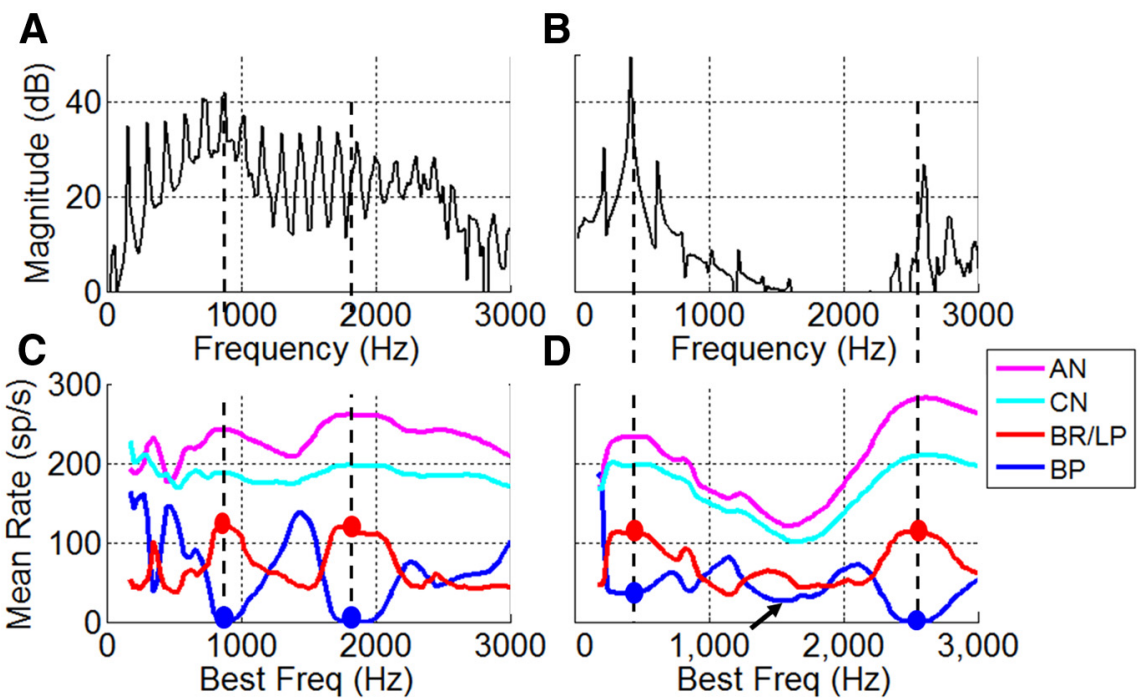

Figure 4 Model IC responses to vowel tokens. $\boldsymbol{A}, \boldsymbol{B}$, The spectra of actual vowels are as follows: /æ/ $(\boldsymbol{A})$, , /i/ $(\boldsymbol{B})$. C, $\boldsymbol{D}, \mathrm{Rate}$ profile of model cells with BP MTFs tuned to F0 (blue) has dips at formants (dots). Rate profile of LPBR model cells with minima in the MTF near F0 (red) has peaks near formants (dots). AN (magenta) and CN/brainstem (cyan) rate profiles. $\boldsymbol{D}$, Rate profile for BP cells has an ambiguous dip (arrow) for vowels with a broad spectral valley. LPBR cells (red) have relatively low rates where spectral energy is low, due to reduced excitatory inputs. Vowel levels were $65 \mathrm{~dB}$ SPL. Model parameters are the same as in Fig. $3 B$.

as opposed to spectral energy or average rates of AN fibers. Model AN rates, shown in response to the vowel /æ/ (had), saturate at moderate-to-high sound levels, obscuring the representations of formant peaks (Fig. 5A). All model responses presented here are based on models for low-threshold high-spontaneous rate AN model fibers, which are the majority of AN fibers (Liberman, 1978). Although responses of medium- or low-spontaneous rate fibers have somewhat larger dynamic ranges and higher thresholds, the representation of formant peaks in all fiber types weakens as the sound level increases and the fibers begin to saturate.

The representations of $\mathrm{F} 1$ and $\mathrm{F} 2$ for the vowel /æ/ (had) in the average discharge rate profiles of populations of model IC cells appear as decreased rates for model BP cells tuned near the formants (Fig. 5B, vertical blue streaks) or increased rates for model LPBR cells (Fig. 5C, vertical orange streaks). The contrast in rates [e.g., the difference between peaks (Fig. 5B,C, red) and minima (Fig. 5B,C, blue)] along the frequency axis varies with SPL. This contrast is strongest for sound levels near $65 \mathrm{~dB}$ SPL (Fig. 5B,C; i.e., in the range of conversational speech). The wide dynamic range of the formant representation is due partly to spike rate adaptation (Dean et al., 2005, 2008; Wen et al., 2009, 2012), which increases the overall dynamic range of auditory neurons, a phenomenon largely explained by the power law synaptic adaptation included in the AN model (Zilany and Carney, 2010).

The reduction in the contrast of rates in the model responses at high levels is consistent with the phenomenon of "rollover," the gradual decrease in speech recognition scores at levels exceeding $80 \mathrm{~dB}$ SPL (Studebaker et al., 1999). The addition of smaller percentages of medium- and low-spontaneous rate AN fibers to the highspontaneous rate model population would slightly in-
A

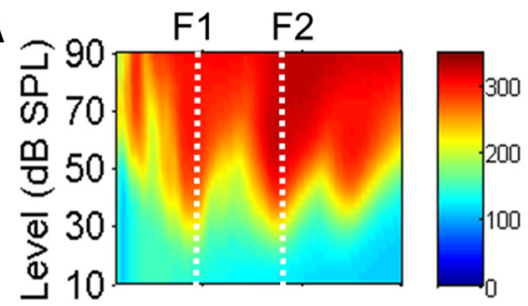

B

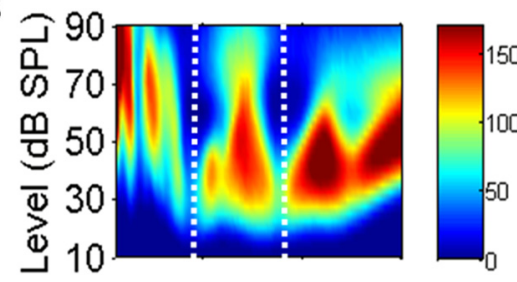

BP

Population

Model

C

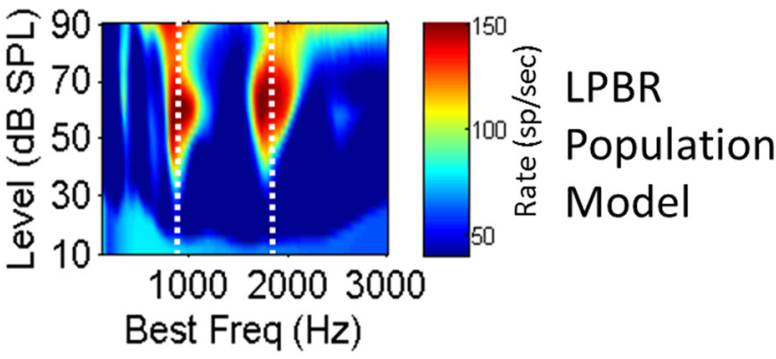

Figure $5 \boldsymbol{A}-\boldsymbol{C}$, Population rate profiles for model AN $(\boldsymbol{A})$, BP $(\boldsymbol{B})$, and LPBR $(\boldsymbol{C})$ cells in response to the vowel /æ/ (had) for a range of sound levels. Vertical dotted lines indicate the first two formant frequencies. $\boldsymbol{A}$, Peaks (red) in AN rates that code formants at low SPLs saturate as level increases. $\boldsymbol{B}$, Dips (blue) in the rate profile for $\mathrm{F} 1$ and $\mathrm{F} 2$ extend from $\sim 30$ to $95 \mathrm{~dB}$ SPL and are strongest at conversational speech levels of 50-70 dB SPL. C, LPBR model cells have peaks (red) in the rate profile at the formant frequencies; these peaks are most robust at conversational speech levels. Model parameters are the same as in Fig. $3 B$. 


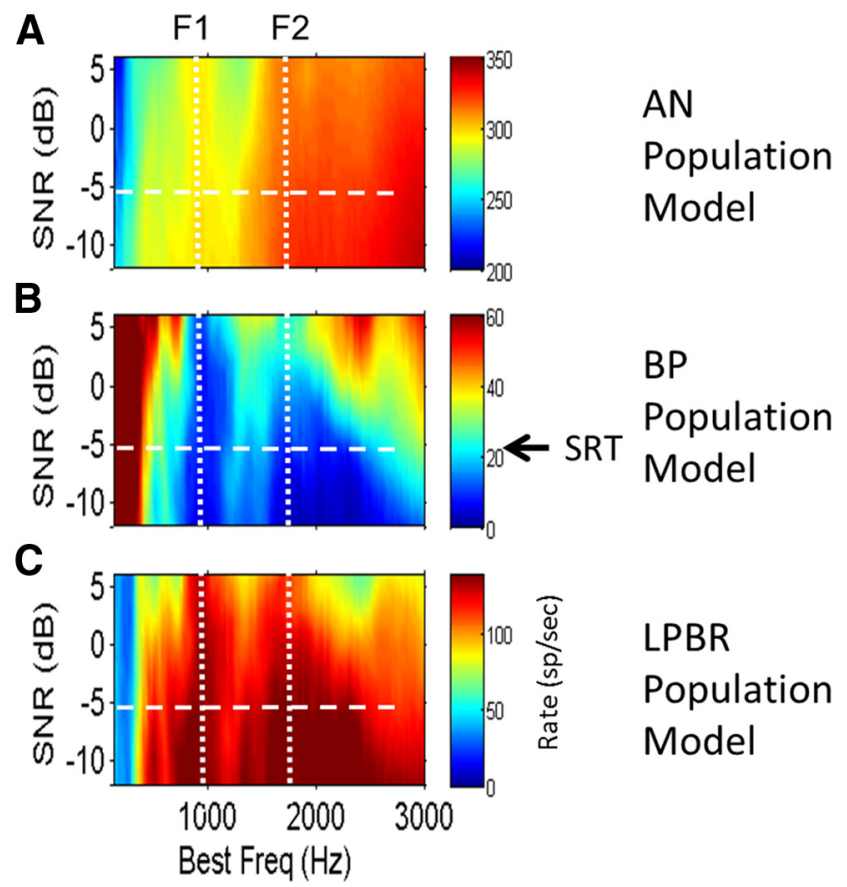

Figure $6 \boldsymbol{A}-\boldsymbol{C}$, Population rate profiles for model AN $(\boldsymbol{A})$, BP $(B)$, and LPBR $(\boldsymbol{C})$ cells in response to the vowel /æ/ (had) for a range of SNRs. Vowel levels were fixed at $65 \mathrm{~dB}$ SPL; the noise level increases toward the bottom of plots. $\boldsymbol{A}$, Saturation of $\mathrm{AN}$ rates by the added noise obscures representations of formant frequencies, especially in the F2 region. $\boldsymbol{B}$, Dips in the average discharge rate profile that indicate the first two formants in the BP population response deteriorate gradually as SNR decreases (toward the bottom of the plot). $\boldsymbol{C}$, Peaks in the rate profile versus SNR for model LPBR cells also deteriorate as SNR decreases. Arrow and horizontal dashed lines indicate the approximate SRT for listeners with normal hearing (Festen and Plomp, 1990). Model parameters are the same as in Fig. 3B.

crease the model dynamic range, but the representation of formants would still roll off at the highest levels (data not shown). The high-spontaneous rate AN models were used as inputs for the IC models shown here to emphasize that the information required for the wide dynamic range of the proposed coding hypothesis is present even in this group of AN fibers, which has the smallest dynamic range.

The representation of formants in the model midbrain average discharge rate profiles is also robust in the presence of additive speech-shaped Gaussian noise across a range of signal-to-noise ratios (SNRs; Fig. 6). Figure 6A shows model AN fibers in response to the vowel /æ/ (had); as SNR decreases, the representation of the formants in the AN discharge rates deteriorates, especially in the F2 frequency region. Formant representation is much more robust in the response profiles of midbrain neurons (Fig. $6 \mathrm{~B}, \mathrm{C})$. The dips in the response profiles of the model BP cells (Fig. 6B) and in the peaks in the LPBR profile (Fig. $6 C)$ deteriorate at approximately the speech reception threshold (SRT), where human listeners have difficulty understanding speech in noise (approximately $-5 \mathrm{~dB}$ SNR; Festen and Plomp, 1990).

\section{Physiological responses}

The vowel-coding model was tested with recordings from IC neurons in awake rabbits in response to 12 contrastive English vowels from one human male speaker with an average F0 of $128 \mathrm{~Hz}$ (Hillenbrand et al., 1995). The responses of 75 neurons with BFs $<4500 \mathrm{~Hz}$ that responded to $65 \mathrm{~dB}$ SPL vowel stimuli were compared to model predictions; a subset of these neurons were also studied at multiple SPLs and SNRs.

Figure 7 illustrates responses of two neurons, one with a BF of $1100 \mathrm{~Hz}$ and a bandpass MTF (Fig. 7A), and the other with a BF of $2000 \mathrm{~Hz}$ and a band-reject MTF (Fig. $7 \mathrm{~B})$. Figure $7, C$ and $D$, shows the average discharge rates for these two cells in response to nine English vowels (black line), along with predictions provided by the BP SFIE (Figs. 1, 4, blue line) and LPBR (Figs. 1, 4, red line) models. For comparison, predictions based on the energy through a gammatone filter centered at the BF are also shown (Figs. 1, 4, green line). The Pearson product moment correlation coefficient between actual rates and each of the predictions is also shown.

The discharge rates of the BP cell were not explained by the stimulus energy near the BF of the neuron. For example, for the cell in Figure $7, A$ and $C$, the energy near the BF is greatest for the vowels / $\mathrm{p} /$ (in "father") and / $/ \mathrm{s} /$ (in "bawd"), yet the discharge rates of the neuron are low in response to these vowels. In contrast, the neuron responds strongly to /i/ (in "heed") and /// (in "hid"), which have relatively low energy near the BF of this neuron (Fig. 7C). The BP SFIE model, however, explains these counterintuitive responses of the BP IC neurons to vowels (Fig. $7 \mathrm{C}$, blue and black lines). The responses of the BP neuron decreased when formant frequencies encroached upon the BF of the neuron $(1300 \mathrm{~Hz})$, as predicted by the SFIE model (Figs. 1F, 4C, blue), because of the reduced rate fluctuations in the those frequency channels. Synchrony capture and saturation of AN fibers tuned near the formant peaks result in reduced rate fluctuations in the responses of those frequency channels. Knowledge of the BF, MTF type, and BMF of the neuron allowed predictions of the vowel responses of the BP cell by the SFIE BP model.

The responses of the band-reject neuron (Fig. 7D, black) increased when formant frequencies were near the BF of the neuron $(2000 \mathrm{~Hz})$, as predicted by the LPBR model (Fig 7D, red). Although the responses of the bandreject neuron were positively correlated to energy near the BF (Fig. 7D, green), the LPBR model responses also reflected trends in the responses of the band-reject neuron that were not explained solely by the stimulus energy in the critical band centered at the BF.

An important property of the proposed model for vowel coding is its resilience across SPL (Fig. 5) and SNR (Fig. 6). Some cells in the IC have discharge rate profiles that are similarly robust across a wide range of stimulus parameters. An example is shown in Figure 8. This neuron had a band-reject MTF (Fig. 8A), and its discharge rates in 
A

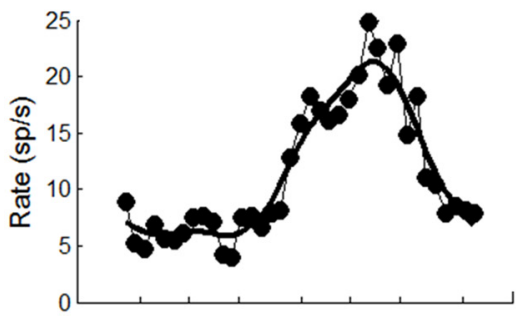

B

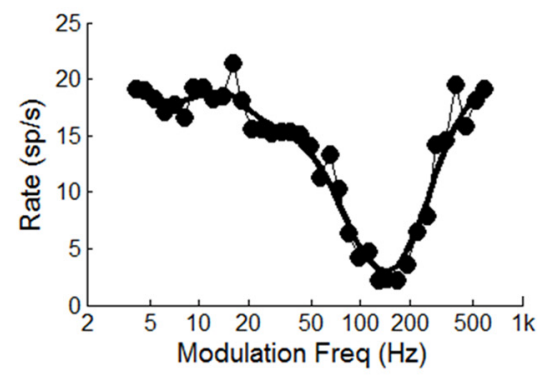

C

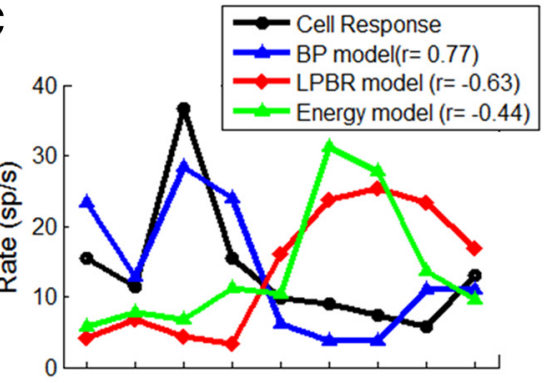

D

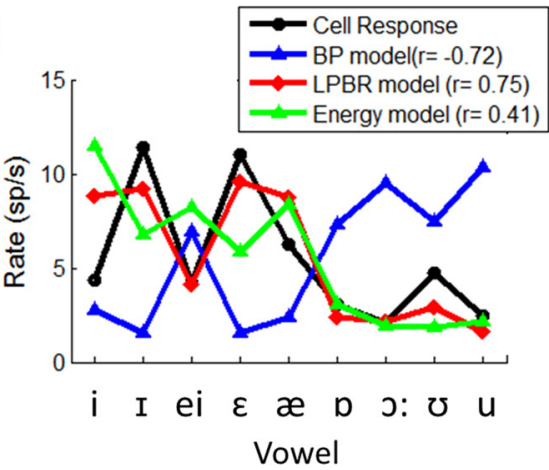

Figure $7 \boldsymbol{A}, \boldsymbol{B}$, Examples of two IC neurons in awake rabbits with bandpass MTF (BF, $1300 \mathrm{~Hz}$; BMF, $130 \mathrm{~Hz}$; $\boldsymbol{A})$ and band-reject MTF $(\mathrm{BF}, 2000 \mathrm{~Hz}$; MTF notch at $150 \mathrm{~Hz} ; \boldsymbol{B})$. C, Black, Average rate of the bandpass neuron in response to nine vowels with F0 = $148 \mathrm{~Hz}$ (Hillenbrand et al., 1995), 65 dB SPL. Blue, Responses of the bandpass SFIE model; red, LPBR model responses; green, energy at the output of a fourth-order gammatone filter at the BF of the cell. Mean and SD of model responses were matched to neural responses. Lines connect the symbols to emphasize patterns in the responses across this set of vowels. $\boldsymbol{D}$, Average rate of the band-reject neuron (black) to vowels with $\mathrm{FO}=95 \mathrm{~Hz}$ presented at $55 \mathrm{~dB}$ SPL, with LPBR model predictions (red), energy (green), and for comparison, the SFIE model response (blue). Model parameters were the same as in Fig. $3 B$.

response to the set of nine vowels presented at $65 \mathrm{~dB}$ SPL were well predicted by the LPBR model and by the energy model (Fig. 8B). The large differences in rate across the set of vowels for this neuron facilitate comparisons of the rate profile across a range of SPLs (Fig. $8 \mathrm{C}$ ) and SNRs (Fig. 8E). As SNR decreases, the rate profile approaches the response to $65 \mathrm{~dB}$ noise alone (Fig. 8E, blue), whereas at high SNRs the profile approaches the response to speech in quiet (Fig. $8 \mathrm{E}$, black). For comparison, responses of a high-spontaneous rate model AN fiber with the same $B F(1100 \mathrm{~Hz})$ are shown for the same range of SPLs (Fig. 8D) and SNRs (Fig. 8E). The AN rates across this set of vowels gradually saturate over this range of sound levels (Fig. 8D). All of the AN responses for stimuli that included the added speech-shaped noise were saturated for the SNRs studied (Fig. 8E).

The physiological results above demonstrate examples of IC responses with features that are consistent with the model. Of 75 neurons that responded to $65 \mathrm{~dB}$ vowel stimuli with $\mathrm{FO}$ in the $100-130 \mathrm{~Hz}$ range, 62 neurons (83\%) had average rates in response to a set of 12 vowels that were significantly correlated (i.e., $r \geq 0.57,2 \mathrm{df}$ ) by at least one of the three models (BP, LPBR, or energy). Of these, $11 \%$ were best predicted by the BP model, and $42 \%$ were best predicted by the LPBR model. Note that many neurons in the IC have more complex MTFs than the simple bandpass and band-reject examples shown above. In particular, MTFs that combine excitatory and inhibitory regions at different modulation frequencies are common
(Krishna and Semple, 2000), and further extension of the model is required to describe the responses of those neurons to vowels. Figure 9 illustrates diverse MTFs, vowel responses, and correlations to model predictions for five additional IC neurons. These complex MTF shapes illustrate the challenge of classifying neurons as simply bandpass or band-reject. Each of these neurons has rates that are enhanced and/or suppressed with respect to the response to the lowest modulation frequency tested. Kim et al. (2015) propose categorization of MTFs as band enhanced or band suppressed, based on comparisons to the response to an unmodulated stimulus. The examples in Figure 9 have responses that are sometimes better predicted by the BP model (Fig. 9A,D), and sometimes by the LPBR model (Fig. 9B,C,E). However, it should be noted that in some cases (Fig. 9A), the correlation between model and neural responses is strongly influenced by the responses to one or two vowels. The correlations in Figure 9 also illustrate that although the LPBR and energy model responses are often highly correlated () , this is not always the case (Fig. 9A,D). In general, for the examples in Figure 9 the BP model provides better predictions of responses for neurons that have peaks in the MTF near the FO of the stimulus, and the LPBR provides better predictions when there is a dip in the MTF near FO. Thus, it is reasonable to hypothesize that quantifying the neural fluctuations established in the periphery near the BF of a neuron, and then applying the features of the MTF at modulation frequencies relevant to the stimulus, will ex- 

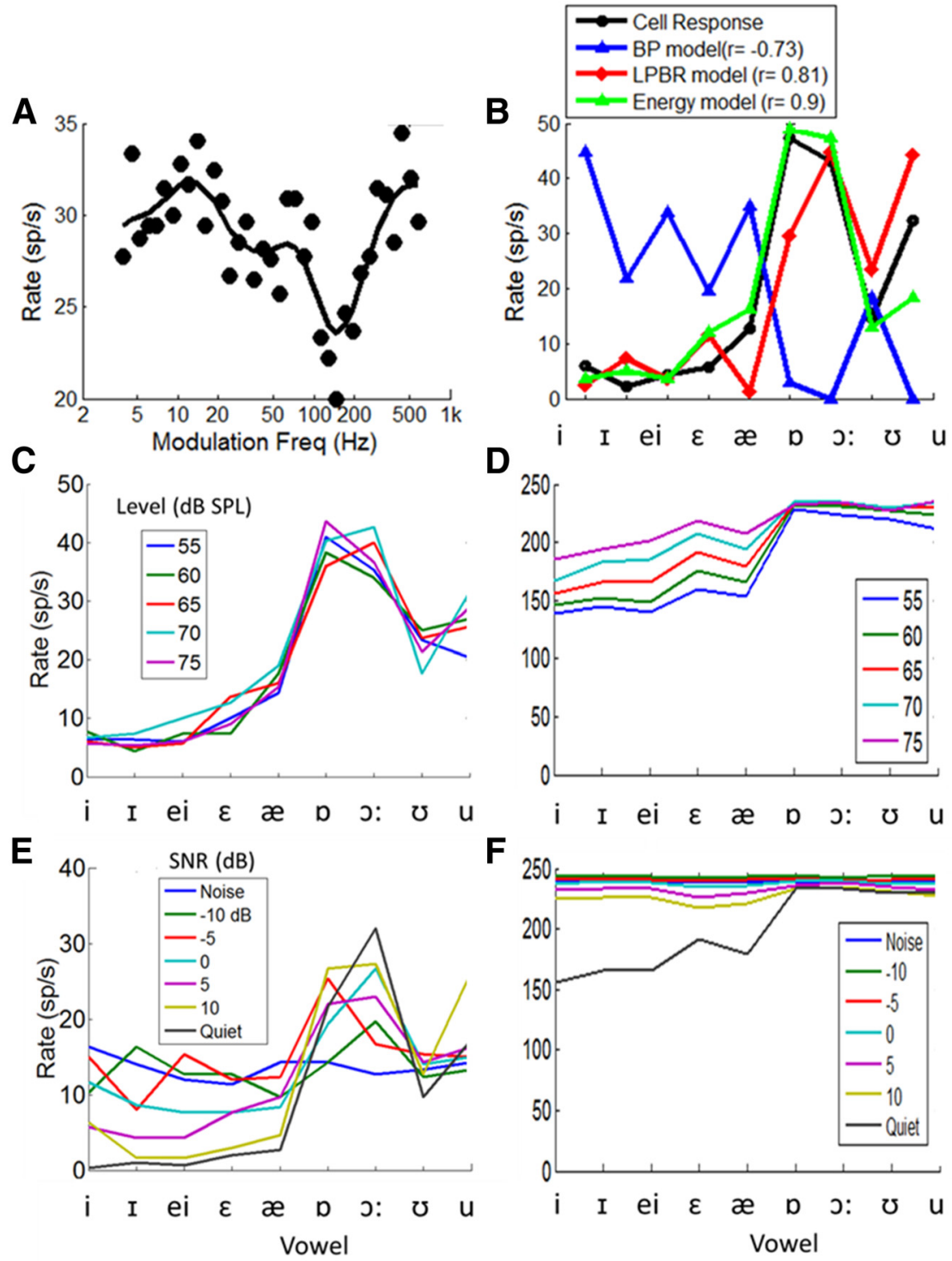

Figure $8 \boldsymbol{A}, \boldsymbol{B}$, Example of a neuron $(\mathrm{BF}, 1100 \mathrm{~Hz})$ with a band-reject MTF $(\boldsymbol{A})$ for which average discharge rates in response to 65 $\mathrm{dB}$ SPL vowels were best predicted by the LPBR model or the energy model $(\boldsymbol{B})$. $\boldsymbol{C}, \boldsymbol{E}$, The patterns of average discharge rates for this neuron across the set of vowels were consistent across a range of SPLs $(\boldsymbol{C})$ and SNRs $(\boldsymbol{E})$. $\boldsymbol{D}, \boldsymbol{F}$, Vowel responses for a model AN fiber with the BF at $1100 \mathrm{~Hz}$ is shown for the same range of SPLs $(\boldsymbol{D})$ and SNRs $(\boldsymbol{F})$. Vowel F0 for all datasets was $95 \mathrm{~Hz}$. IC Model parameters were the same as in Fig. $3 B$.

plain the vowel responses for cells with complex MTFs. This strategy provides a novel and general framework for understanding how complex sounds with strong fluctuations, such as voiced speech, are encoded at the level of the midbrain.

\section{Discussion}

Previous studies of the neural representation of vowels have largely focused on the coding of spectral energy by the AN (Liljencrants and Lindblom, 1972; Delgutte and Kiang, 1984; Young, 2008; Lindblom et al., 2009). Codes based on AN average discharge rates and/or temporal fine structure are problematic because of limited dynamic range and background noise. Many AN models, especially those used in the phonetics literature, are based on linear filter banks (Lindblom, 1990; Diehl and Lindblom, 2004; Ghosh et al., 2011). The model presented here, in contrast, includes the nonlinear attributes of AN responses, including level-dependent tuning bandwidth, synchrony capture, and saturation, all of which influence the neural fluctuations in response to speech sounds that ultimately project to the IC. The coding hypothesis here focuses on the F0-related fluctuations in the AN responses and how they vary across the population. These fluctuations are important because IC neurons are tuned to both audio and modulation frequencies. This tuning provides sensitivity to the contrast in low-frequency, pitch-related fluctuations across a population of neurons with different best frequencies (Fig. 1B,C). 

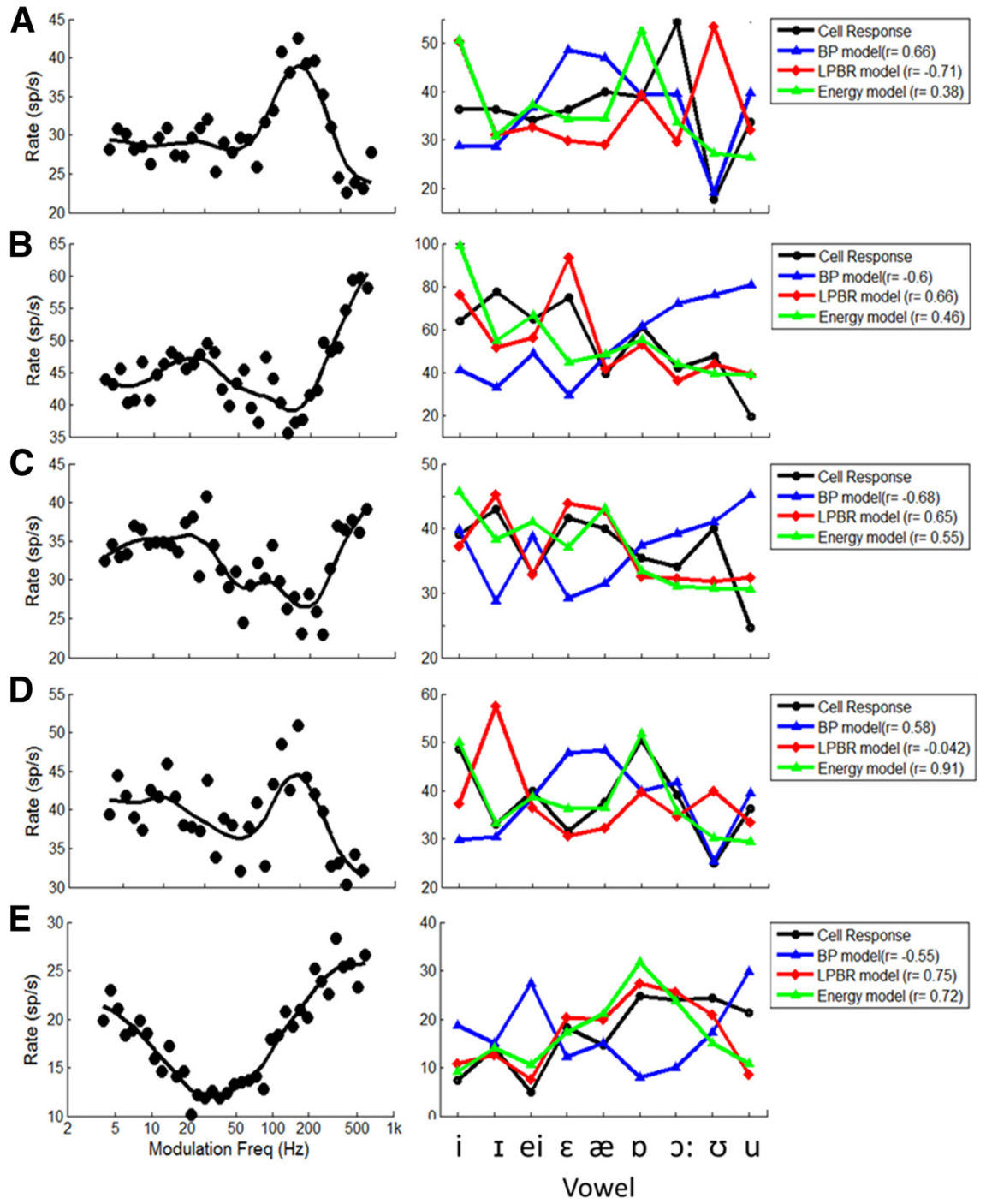

Figure 9 Example of five neurons with diverse MTFs (left panels) and predictions of responses to nine English vowels (right panels) at $65 \mathrm{~dB}$ SPL, with correlations to the model predictions in the legends. $\boldsymbol{A}-\boldsymbol{E}$, BFs were $3900 \mathrm{~Hz}(\boldsymbol{A}), 2700 \mathrm{~Hz}(\boldsymbol{B}), 1900 \mathrm{~Hz}(\boldsymbol{C}), 4020$ $\mathrm{Hz}(\boldsymbol{D})$, and $1485 \mathrm{~Hz}(\boldsymbol{E})$. Model parameters were the same as in Fig. $3 B$.

IC responses tuned to a range of modulation frequencies encode vowel formant frequencies across a range of pitches, capturing an essential aspect of speech (Diehl, 2008).

This model framework provides a context for understanding several general questions related to vowel systems, which exhibit universal properties that generalize across languages. For example, formant locations appear to disperse optimally within an F1-F2 space, referred to as dispersion theory (Liljencrants and Lindblom, 1972; Lindblom, 1986; Schwartz et al., 1997; Diehl and Lindblom, 2004). This dispersion results in consistencies among linguistic vowel systems in the appearance of vowel contrasts as vowel systems increase in size. Our model for neural representations of vowels thus provides a new tool for understanding how the role of the auditory system in shaping vowel systems.

This model also provides a framework to study the relative spacing of formant peaks, F1, F2, and F3, which define single vowels. The neural resolution for coding separate formants, or for merging them, depends upon both the separation of the formant peaks and the widths of the formant bands. Limits in neural resolution along the frequency axis for coding single-formant peaks would determine when nearby formants merge perceptually (Chistovich and Lublinskaya, 1979). This concept underlies focalization-dispersion theory (Schwartz et al, 1997; Becker-Kristal, 2010). In the neural model, the representation of the width of a single formant along the frequency axis (Figs. 1F, 1G, 2, 6) depends upon the width of the modulation transfer functions for these neurons (Figs. $1 \mathrm{D}, \mathrm{E}, 4)$. Future studies to test the hypothesis presented here should include synthetic vowel sounds, in which the key parameters can be systematically manipulated with respect to the frequency and modulation tuning of a given neuron, as well as higher-level features such as formant spacing. These stimuli would also provide stronger sta- 
tistical tests of the correlations between model and neural responses than was possible with the small set of spoken vowels used in this study.

Recent results in the cortex suggest that phonetic features are encoded in the responses of the superior temporal gyrus (Mesgarani et al., 2008; Pasley et al., 2012), but the problem of how neural maps at cortical levels are created from the acoustic stimulus remains. The results presented here suggest a framework for subcortical neural coding of phonetic features based on patterns of F0-related neural fluctuations. These patterns are established in the auditory periphery by the nonlinear response properties of inner ear mechanics and sensory transduction. Contrasts in these patterns are then enhanced by the sensitivity of midbrain neurons to fluctuation frequencies. The potential also exists for amplification of these contrasts in the thalamus and cortex by interactions between inputs from bandpass and band-reject midbrain responses. Responses of midbrain cells with complex MTFs, characterized by a combination of inhibition and excitation (Krishna and Semple, 2000), could serve as effective "edge-detectors," further enhancing the contrasts in rate fluctuations across the neural population. In general, the combination of spectral frequency tuning and modulation frequency processing in the midbrain provides a substrate for parsing complex sounds into the features that are required for higher-level phonetic representations.

The stimuli modeled and tested in this study were limited to vowels, and the voiced structure of these sounds has a strong influence on the responses. It is interesting to consider how the properties of these neurons would influence responses to other types of speech sounds. Unvoiced vowels exist in whispered speech, and in vocoded speech, such as that used in cochlear implant simulations (Shannon et al., 1995), as well as conditioned alternates of vowels in several languages (Ladefoged and Maddieson, 1996). Unvoiced or devoiced vowels have reduced intelligibility compared to normal vowels. The model presented here would respond mainly to the energy profile in unvoiced vowels, such that formants would be coded by increased rates for neurons tuned near formants. These energy-related responses would be correlated with the representation of voiced vowels in the LPBR model, though with reduced contrast in rate as a function of frequency. Consonants represent another diverse and important set of speech sounds, sets of which (obstruents) are commonly voiceless ( $\mathrm{p}, \mathrm{t}, \mathrm{k}, \mathrm{ch}$ ), and sometimes are characterized by a noisy source (e.g., fricatives; Stevens, 1998; Ladefoged, 2006). Similar to vowels, the consonants set up a pattern of neural fluctuations in the peripheral response that will ultimately drive the responses at the level of the midbrain. Future studies will extend the general approach presented here to include a larger set of speech sounds. An interesting question is how midbrain neurons with different MTFs will represent the slopes and peaks in consonant spectra, which result in nonperiodic but potentially strong fluctuations in peripheral responses.

The vowel-coding hypothesis presented here has implications for several applications related to speech pro- cessing. Accurate formant identification in the presence of substantial background noise is critical for automatic speech recognition systems, yet is difficult to achieve. The hypothesis also provides a new framework for speech enhancement algorithms for listeners with and without hearing loss. The code is substantially affected by common aspects of hearing loss, such as broadened frequency tuning in the inner ear, which distorts the representation of both the spectrum and amplitude modulations. The proposed code is also affected by changes in synchrony capture that would accompany hearing loss. Loss of synchrony capture has a profound effect on the nature of the neural fluctuations in peripheral responses, and thus on the responses of central neurons that are driven by these fluctuations. The hypothesis thus informs the development of algorithms for new hearing aids and cochlear implant speech processors that encode information in temporal envelopes.

\section{References}

Becker-Kristal R (2010) Acoustic typology of vowel inventories and dispersion theory: insights from a large cross-linguistic corpus. $\mathrm{PhD}$ thesis, UCLA.

Blackburn CC, Sachs MB (1990) The representations of the steadystate vowel sound /e/ in the discharge patterns of cat anteroventral cochlear nucleus neurons. J Neurophysiol 63: 1191-1212.

Chistovich LA, Lublinskaya VV (1979) The "center of gravity" effect in vowel spectra and critical distance between the formants: psychoacoustical study of the perception of vowel-like stimuli. Hear Res 1:185-195. CrossRef

Dean I, Harper NS, McAlpine D (2005) Neural population coding of sound level adapts to stimulus statistics. Nat Neurosci 8:16841689. CrossRef Medline

Dean I, Robinson BL, Harper NS, McAlpine D (2008) Rapid neural adaptation to sound level statistics. J Neurosci 28:6430-6438. CrossRef Medline

Delgutte B, Hammond BM, Cariani PA (1998) Neural coding of the temporal envelope of speech: relation to modulation transfer functions. In: Psychophysical and physiological advances in hearing (Palmer AR, Summerfield AQ, Meddis R, eds), pp 595-603. London, UK: Whurr Publishers.

Delgutte B, Kiang NY (1984) Speech coding in the auditory nerve: I. Vowel-like sounds. J Acoust Soc Am 75:866-878. Medline

Deng L, Geisler CD (1987) Responses of auditory-nerve fibers to nasal consonant-vowel syllables. J Acoust Soc Am 82:1977-1988. Medline

Diehl R, Lindblom B (2004) Explaining the structure of feature and phoneme inventories: the role of auditory distinctiveness. In: Speech processing in the auditory system (Greenberg S, Ainsworth WA, Popper AN, Fay RR, eds), pp 101-162. New York: Springer.

Diehl RL (2008) Acoustic and auditory phonetics: the adaptive design of speech sound systems. Philos Trans R Soc Lond B Biol Sci 363:965-978. CrossRef Medline

Fant G (1960) Acoustic theory of speech production. Hague, The Netherlands: Mouton.

Festen JM, Plomp R (1990) Effects of fluctuating noise and interfering speech on the speech-reception threshold for impaired and normal hearing. J Acoust Soc Am 88:1725-1736. Medline

Frisina RD, Smith RL, Chamberlain SC (1990) Encoding of amplitude modulation in the gerbil cochlear nucleus: I. A hierarchy of enhancement. Hear Res 44:99-122. CrossRef

Gai Y, Carney LH (2008) Influence of inhibitory inputs on rate and timing of responses in the anteroventral cochlear nucleus. J Neurophysiol 99:1077-1095. CrossRef Medline 
Ghosh PK, Goldstein LM, Narayanan SS (2011) Processing speech signal using auditory-like filterbank provides least uncertainty about articulatory gestures. J Acoust Soc Am 129:4014-4022. CrossRef Medline

Grothe B, Park TJ, Schuller G (1997) Medial superior olive in the free-tailed bat: response to pure tones and amplitude-modulated tones. J Neurophysiol 77:1553-1565. Medline

Hillenbrand J, Getty LA, Clark MJ, Wheeler K (1995) Acoustic characteristics of American English vowels. J Acoust Soc Am 97:30993111. Medline

Joris PX, Schreiner CE, Rees A (2004) Neural processing of amplitude-modulated sounds. Physiol Rev 84:541-577. CrossRef Medline

Joris PX, Yin TC (1998) Envelope coding in the lateral superior olive. III. Comparison with afferent pathways. J Neurophysiol 79:253269. Medline

Kim DO, Zahorik P, Carney LH, Bishop BB, Kuwada S (2015) Auditory distance coding in rabbit midbrain neurons and human perception: monaural amplitude modulation depth as a cue. J Neurosci 35:5360-5372. CrossRef Medline

Krishna BS, Semple MN (2000) Auditory temporal processing: responses to sinusoidally amplitude-modulated tones in the inferior colliculus. J Neurophysiol 84:255-273. Medline

Ladefoged P (2006) A course in phonetics. Belmont, CA: Thomson Wadsworth.

Ladefoged P, Maddieson I (1996) Sounds of the world's languages. Hoboken, NJ: Wiley-Blackwell.

Langner G (1992) Periodicity coding in the auditory system. Hear Res 60:115-142. Medline

Liberman MC (1978) Auditory-nerve response from cats raised in a low-noise chamber. J Acoust Soc Am 63:442-455. Medline

Liljencrants J, Lindblom B (1972) Numerical simulation of vowel quality systems: the role of perceptual contrast. Language 48:839862. CrossRef

Lindblom B (1986) Phonetic universals in vowel systems. In: Experimental phonology (Ohala J, Jaeger J, eds), pp 13-44. Orlando, FL: Academic.

Lindblom B (1990) Explaining phonetic variation: a sketch of the $\mathrm{H}$ \& $\mathrm{H}$ theory. In: Speech production and speech modeling (Hardcastle WJ, Marchal A, eds), pp. 403-439. Dordrecht, The Netherlands: Kluwer.

Lindblom B, Diehl R, Creeger C (2009) Do "dominant frequencies" explain the listener's response to formant and spectrum shape variations? Speech Comm 51:622-629. CrossRef Medline

Mesgarani N, David SV, Fritz JB, Shamma SA (2008) Phoneme representation and classification in primary auditory cortex. J Acoust Soc Am 123:899-909. CrossRef Medline

Miller RL, Schilling JR, Franck KR, Young ED (1997) Effects of acoustic trauma on the representation of the vowel "eh" in cat auditory nerve fibers. J Acoust Soc Am 101:3602-3616. Medline

Nelson PC, Carney LH (2004) A phenomenological model of peripheral and central neural responses to amplitude-modulated tones. J Acoust Soc Am 116:2173-2186. Medline

Nelson PC, Carney LH (2007) Neural rate and timing cues for detection and discrimination of amplitude-modulated tones in the awake rabbit inferior colliculus. J Neurophysiol 97:522-539. CrossRef Medline
Pasley BN, David SV, Mesgarani N, Flinker A, Shamma SA, Crone NE, Knight RT, Chang EF (2012) Reconstructing speech from human auditory cortex. PLoS Biol 10:e1001251. CrossRef Medline

Sachs MB, Abbas PJ (1974) Rate versus level functions for auditorynerve fibers in cats: tone-burst stimuli. J Acoust Soc Am 56:18351847. Medline

Sachs MB, Young ED (1979) Encoding of steady-state vowels in the auditory nerve: representation in terms of discharge rate. J Acoust Soc Am 66:470-479. CrossRef

Schilling JR, Miller RL, Sachs MB, Young ED (1998) Frequencyshaped amplification changes the neural representation of speech with noise-induced hearing loss. Hear Res 117:57-70. Medline

Schmitzer-Torbert N, Jackson J, Henze D, Harris K, Redish AD (2005) Quantitative measures of cluster quality for use in extracellular recordings. Neuroscience 131:1-11. CrossRef Medline

Schwartz JL, Boë LJ, Vallée N, Abry C (1997) The dispersionfocalization theory of vowel systems. J Phon 25:255-286. CrossRef

Schwarz DM, Zilany MS, Skevington M, Huang NJ, Flynn BC, Carney LH (2012) Semi-supervised spike sorting using pattern matching and a scaled Mahalanobis distance metric. J Neurosci Methods 206:120-131. CrossRef Medline

Shannon RV, Zeng FG, Kamath V, Wygonski J, Ekelid M (1995) Speech recognition with primarily temporal cues. Science 270 : 303-304. Medline

Stevens K (1998) Acoustic phonetics. Cambridge, MA: MIT.

Studebaker GA, Sherbecoe RL, McDaniel DM, Gwaltney CA (1999) Monosyllabic word recognition at higher-than-normal speech and noise levels. J Acoust Soc Am 105:2431-2444. Medline

Wen B, Wang GI, Dean I, Delgutte B (2009) Dynamic range adaptation to sound level statistics in the auditory nerve. J Neurosci 29:13797-13808. CrossRef Medline

Wen B, Wang GI, Dean I, Delgutte B (2012) Time course of dynamic range adaptation in the auditory nerve. J Neurophysiol 108:69-82. CrossRef Medline

Yates GK (1990) Basilar membrane nonlinearity and its influence on auditory nerve rate-intensity functions. Hear Res 50:145-162. Medline

Yates GK, Winter IM, Robertson D (1990) Basilar membrane nonlinearity determines auditory nerve rate-intensity functions and cochlear dynamic range. Hear Res 45:203-219. Medline

Young ED (2008) Neural representation of spectral and temporal information in speech. Philos Trans R Soc Lond B Biol Sci 363: 923-945. CrossRef Medline

Young ED, Sachs MB (1979) Representation of steady-state vowels in the temporal aspects of the discharge patterns of populations of auditory-nerve fibers. J Acoust Soc Am 66:1381-1403. Medline

Zilany MS, Bruce IC, Nelson PC, Carney LH (2009) A phenomenological model of the synapse between the inner hair cell and auditory nerve: long-term adaptation with power-law dynamics. $J$ Acoust Soc Am 126:2390-2412. CrossRef Medline

Zilany MS, Carney LH (2010) Power-law dynamics in an auditorynerve model can account for neural adaptation to sound-level statistics. J Neurosci 30:10380-10390. CrossRef Medline

Zilany MS, Bruce IC, Carney LH (2014) Updated parameters and expanded simulation options for a model of the auditory periphery. J Acoust Soc Am 135:283-286. CrossRef Medline 